\title{
Treatment of Chronic Isolated Finger Flexor Tenosynovitis Through 50\% Dehydrated Alcohol Installation
}

\author{
Jae Eun Shin, $\mathrm{MD}^{1}$, Jung Hyun Park, MD${ }^{1}$, Ho Sung Yi, MD², Byung Kook Ye, MD², Hyoung Seop Kim, MD² \\ ${ }^{1}$ Department of Rehabilitation Medicine and Rehabilitation Institute of Neuromuscular Disease, \\ Yonsei University College of Medicine, Seoul; ${ }^{2}$ Department of Rehabilitation Medicine, \\ National Health Insurance Corporation Ilsan Hospital, Goyang, Korea
}

The isolated idiopathic finger flexor tenosynovitis is a rare condition, related to diversed etiologies. The traditional treatment of flexor tenosynovitis includes medications and injection of steroids. If the conservative treatment is not effective, surgical management is usually recommended. And alcohol installations have been rarely performed. We are reporting an extremely rare case of a 56-year-old man who had chronic idiopathic isolated finger flexor tenosynovitis which was treated through alcohol injections. The patient had not yet been treated despite of medication and serial injections of steroid. We performed $1 \mathrm{~mL}$ of $50 \%$ ethanol injection for the initial treatment and the second injection was done in the same way 10 months later due to the improvements of the patient's clinical symptoms and images of the follow-up ultrasonography. As a result, the authors suggest alcohol installation as an alternative non-surgical treatment for flexor tenosynovitis when other conservative managements are not effective enough.

Keywords Tenosynovitis, Alcohols, Ultrasonography

\section{INTRODUCTION}

Digital flexor tenosynovitis may be caused by various inflammatory diseases such as tuberculosis, sarcoid, collagen-vascular disease, rheumatoid arthritis, and chronic

\footnotetext{
Received April 19, 2012; Accepted September 20, 2012 Corresponding author: Hyoung Seop Kim

Department of Rehabilitation, National Health Insurance Corporation Ilsan Hospital, 100 Ilsan-ro, Ilsandong-gu, Goyang 410-719, Korea Tel: +82-31-900-0137, Fax: +82-31-900-0343, E-mail: rekhs@nhimc.or.kr

(c) This is an open-access article distributed under the terms of the Creative Commons Attribution Non-Commercial License (http://creativecommons. org/licenses/by-nc/3.0) which permits unrestricted noncommercial use, distribution, and reproduction in any medium, provided the original work is properly cited.

Copyright ( 2013 by Korean Academy of Rehabilitation Medicine
}

mycotic infections. Chronic inflammatory diseases such as leukemia, external trauma, and repetitive use of fingers and pregnancy are also a cause of distal flexor tenosynovitis [1-3].

Physiotherapy, anti-inflammatory pharmacotherapy and steroid injections under ultrasound guidance are conservative treatments for chronic flexor tenosynovitis. If conservative treatments are ineffective, the tenosynovectomy can be performed. Recently, treatments for platelet-derived growth factors such as injection for growth factors, anti-neovascular destruction through polidocanol injection or electrocoagulation and extracorporeal shock wave have been cited as new treatments for flexor tenosynovitis [4].

This is to report a patient, with chronic isolated digital 
flexor tenosynovitis caused by non-specific inflammatory responses, who was refractory to synovial cyst steroid injection but was cured with alcohol injected into the tendon sheath.

\section{CASE REPORT}

A 56-year-old man visited the rehabilitation department at our hospital with major complaints of painful swelling on his right forefinger. The patient was an office worker and had no history of external trauma. He indicated ' 2 ' on the visual analogue scale (VAS) in ordinary position but indicated ' 4 ' during finger extensions. On physical examination, tenderness-free edema was observed on his right forefinger, but there were no suspected infections e.g., localized burning sensation or redness. According to manual muscle testing using the Medical Research Council scale for muscular strength, the right forefinger measured 5 during both flexion and extension. Abnormalities were not observed in the metacarpophalangeal joint during the right forefinger extension, but its flexion angle did not exceed 30 degrees.

The patient had taken hypoglycemic drugs due to diabetes, but had no past medical histories related to gout, arthritis, and tuberculosis. He tested negative on a tuberculosis polymer chain reaction test and chest radiography performed at another hospital. He underwent a blood test after visiting this hospital, and white blood cells, red blood cells, erythrocyte sedimentation rate (ESR), and Creactive protein (CRP) were all within normal levels. He was also tested negative for anti-cyclic citrullinated peptide, rheumatoid factor, and antinuclear antibodies.

On the longitudinal sectional image of his magnetic resonance imaging (MRI) scan performed at another hospital, synovial fluid retention was observed and the flexor tendon had increased in cross-sectional area, which was almost a positive proof of flexor tenosynovitis (Fig. 1A). On the transverse image, the synovial cyst significantly increased in cross-sectional area (Fig. 1B). He had had such symptoms for 9 months before visiting this hospital. Pharmacotherapy and physiotherapy were performed for 4 months and steroid was injected 3 times, but there were no improvements in symptoms.

Simple radiography, performed at this hospital, did not show noteworthy abnormalities, but ultrasonography iU22 (Philips Medical System, Bothell, WA, USA) showed proliferations of blood cells in the right forefinger on both the longitudinal section and the transverse section. In addition, for hyperechoic characteristics, profuse synovial fluid retention and flexor thickening were noted through ultrasonography (Fig. 2A, B). Power Doppler ultrasonography showed blood being supplied to the proliferated synovial cells (Fig. 2C).

Under ultrasonography guidance, $1 \mathrm{~mL}$ of $50 \%$ ethanol was injected into the tendon sheath of the proximal interphalangeal joint of the forefinger where blood flow had
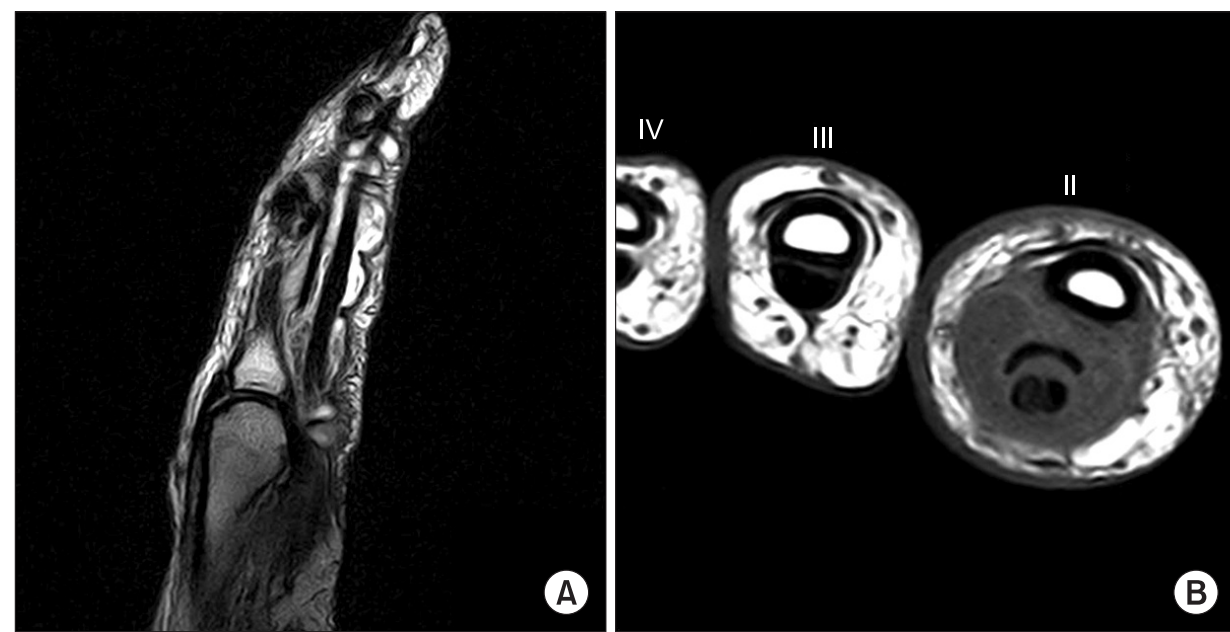

Fig. 1. Magnetic resonance images of right index finger. (A) Sagittal T2-weighted image showing accumulation of fluid around flexor tendon with lobulated margin and thickening of tendon, without definite abnormalities in the adjacent bones. (B) Note the reactive diffuse thickening of visceral layer of tendon sheath and synovial effusion in the right index finger (II) and the cross-sectional size of the index finger (II) is much larger relative to that of the middle finger (III). 
increased. The patient did not show side effects excluding pain at the injection site.

On the second hospital visit (a month later), the VAS score fell to 1 even during joint movements and the range of joint motion approached 60 degrees. However, swelling remained.

On the third hospital visit (10 months later), the range of joint motion was the same as before. The VAS score had risen to 2 and edema was persistently visible. An additional alcohol injection was performed as before.

On the fourth hospital visit (12 months later), he indi- cated 'zero' on VAS during rest and during flexion and extension of the metacarpophalangeal joint of the right forefinger. On physical examination, the flexion angle approached 70 degrees and tenderness on the forefinger did not occur. In addition, edema had subsided significantly.

On the fifth hospital visit (22 months after the first alcohol injection), the VAS score was still 'zero' and edema had subsided remarkably from the previous 10 months (the fourth hospital visit). On physical examination, he did not show any pains during movement of the metacarpophalangeal joint of the right forefinger and could flex it
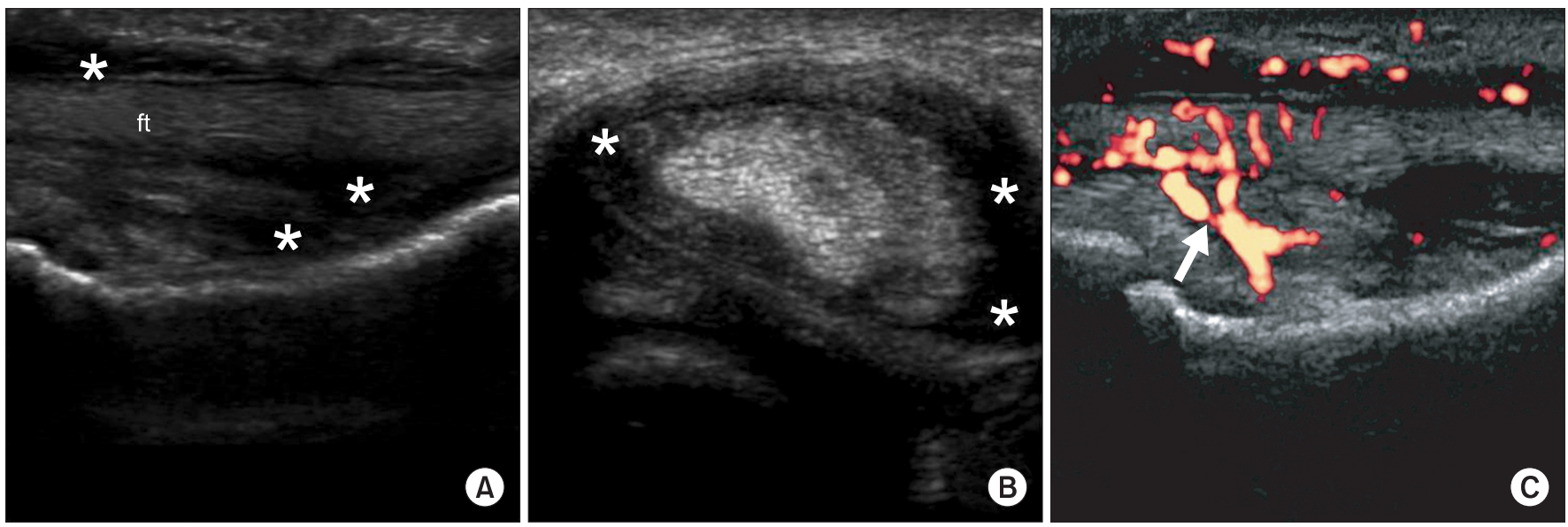

Fig. 2. Pretreatment ultrasonography images of chronic right index finger swelling. (A) Longitudinal image of the right index finger demonstrated a flexor tendon sheath distended by echogenic synovial fluid effusion (asterisks) surrounding the flexor tendon (ft). (B) Sagittal image of the right index finger showed synovial effusion (asterisks). (C) Power Doppler sonography image showed increased blood flow (arrow) within the synovial sheath.
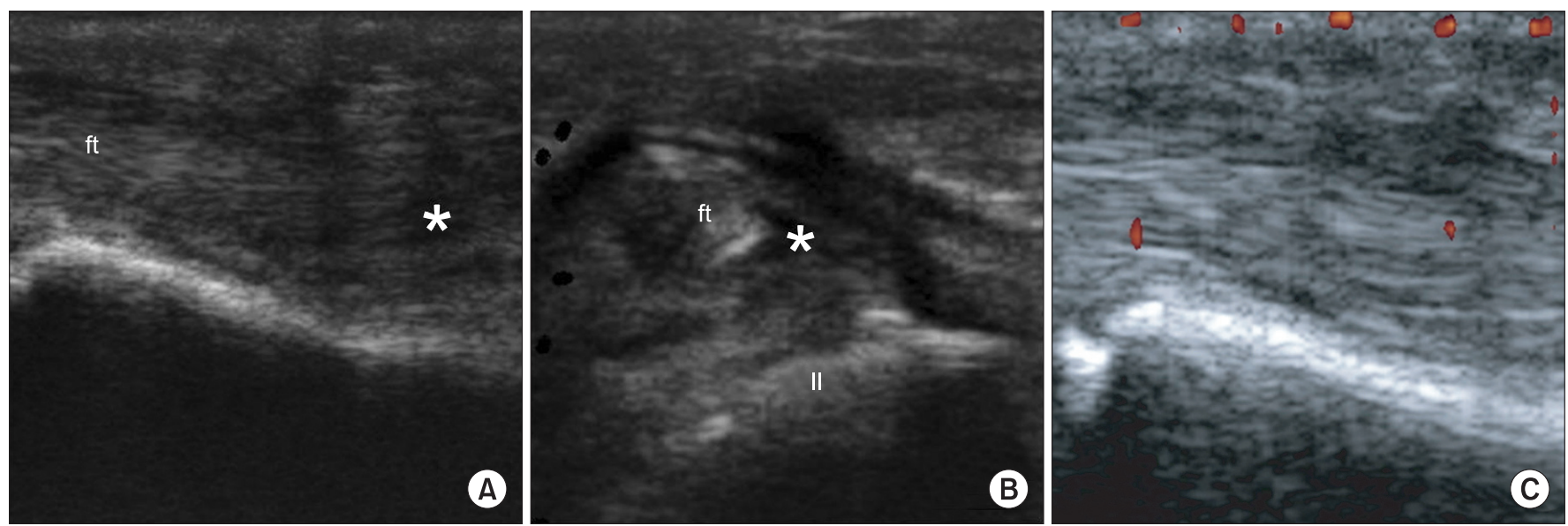

Fig. 3. Follow-up ultrasonography images of right index finger taken 22 months later after 1st injection. (A) Longitudinal images shows synovial fluid effusion (asterisk) of the right index finger is clearly decreased. (B) Sagittal images of the right index finger (II) shows synovial effusions are significantly reduced. (C) Power Doppler sonography image shows the disappearance of blood flow in synovial sheath, compared with previous findings. 
90 degrees.

Ultrasonography showed that synovial cells had proliferated and nutrition was being provided from blood vessels (Fig. 3). Two alcohol injections did not cause noteworthy complications during the follow-ups conducted for 22 months.

\section{DISCUSSION}

Flexor tenosynovitis is the inflammation of the fluidfilled sheath that surrounds a tendon, i.e., the synovium. A flexor tendon, during chronic inflammation, may cause painful edema, and long-term degeneration resulting in knuckle pads or rarely flexor tendon ruptures $[4,5]$.

For chronic flexor tenosynovitis, conservative treatment is performed without surgical treatments, and thus, it cannot be diagnosed pathologically and difficult to identify the cause in most cases. Since simple radiography is not sufficient, MRI or ultrasonography is necessary. In addition, ESR and CRP may be within the normal levels in the case of a mild bacterial infection and thus blood tests are not sufficient for diagnosis. This blood results may be due to non-specific inflammatory responses and rheumatic or tubercular tenosynovitis which comprise a higher percentage in the cases that have been reported so far [6]. In particular, tubercular tenosynovitis tends to be slow in progress, and moreover, it is difficult to distinguish from rheumatic tenosynovitis or inflammatory responses caused by non-specific infections [7].

Generally, the synovial sheath situated on the flexor tendon is not easily observed, even on high-resolution ultrasonography. But in the case of acute flexor tenosynovitis, the surrounding tissues, which are affected by the synovium expansion, enlarges due to hypoechoic or anechoic collections. During the acute phase, a hyperemic flow signal is observed around the synovial fold on color Doppler ultrasonography. In subacute or chronic flexor tenosynovitis, the synovial sheath tends to be thicker [8].

In many cases, steroid injections are used against nonspecific isolated flexor tenosynovitis, but there are controversies about the mechanisms and effectiveness $[2,4]$.

It is difficult to treat chronic flexor tenosynovitis due to synovial cell proliferations and angioplasia $[4,9]$. In case it does not take a favorable turn after conservative treatments, the synovial cyst is removed surgically [4].

As for chronic flexor tenosynovitis against which con- servative treatment is not efficacious, alcohol is injected under ultrasound guidance, which removes proliferated synovial cells by protein denaturation [9]. The alcohol injection is cheaper than surgical treatments, causes less complications, and has the merit of rapid recovery $[4,10]$.

So far, alcohol has been used for the treatment of vascular malformation. In cases where $95 \%$ alcohol is injected to treat venous malformation, the nerves or tissues may be injured, but serious side effects have not yet been reported. Although patients may feel pain after being injected, it does not matter much as alcohol may block the nerves $[9,10]$. In this case where the patient was injected with comparatively low concentration (50\%) and small quantity (1 mL) of alcohol, less safety issues are likely to occur. In principle, however, it should be performed only in cases where conservative treatment is not efficacious [10].

Non-specific chronic flexor tenosynovitis is a rare occurrence, and further, there have been few cases where it was treated by alcohol injections. This report is expected to be helpful in diagnosing and treating chronic isolated flexor tenosynovitis.

\section{CONFLICT OF INTEREST}

No potential conflict of interest relevant to this article was reported.

\section{REFERENCES}

1. Klug JD. MR diagnosis of tenosynovitis about the wrist. Magn Reson Imaging Clin N Am 1995;3:305-12.

2. McGrath MH. Local steroid therapy in the hand. J Hand Surg Am 1984;9:915-21.

3. Al-Qattan MM, Al-Namla A, Al-Thunayan A, AlOmawi M. Tuberculosis of the hand. J Hand Surg Am 2011;36:1413-21.

4. Maffulli N, Longo UG, Loppini M, Denaro V. Current treatment options for tendinopathy. Expert Opin Pharmacother 2010;11:2177-86.

5. Rosenthal EA. Tenosynovitis: tendon and nerve entrapment. Hand Clin 1987;3:585-609.

6. Jung JM, Kim JO, Yun YH, Koh YD, Yoo JD, Jung DH, et al. Tenosynovectomy for chronic flexor tenosynovitis at the wrist. J Korean Soc Surg Hand 2004;9:83-8.

7. Bush DC, Schneider LH. Tuberculosis of the hand and 
wrist. J Hand Surg Am 1984;9:391-8.

8. Bianchi S, Martinoli C. Ultrasound of the musculoskeletal system. Heidelberg: Springer; 2007.

9. Shin JB, Joo SH, Kim HS, Cho HK. Treatment of synovial proliferative subdeltoid bursitis through alcohol installation: a case report. J Korean Acad Rehabil Med 2010;34:106-9.

10. Deveikis JP. Percutaneous ethanol sclerotherapy for vascular malformations in the head and neck. Arch Facial Plast Surg 2005;7:322-5. 